\title{
A perfectly conducting surface in electrodynamics with Lorentz symmetry breaking
}

\author{
L. H. C. Borges ${ }^{1, \mathrm{a}}$, F. A. Barone ${ }^{2, \mathrm{~b}}$ \\ ${ }^{1}$ UNESP, Campus de Guaratinguetá, DFQ, Avenida Dr. Ariberto Pereira da Cunha 333, CEP 12516-410 Guaratinguetá, SP, Brazil \\ ${ }^{2}$ IFQ, Universidade Federal de Itajubá, Av. BPS 1303, Pinheirinho, Caixa Postal 50, Itajubá, MG 37500-903, Brazil
}

Received: 3 July 2017 / Accepted: 5 October 2017 / Published online: 20 October 2017

(C) The Author(s) 2017. This article is an open access publication

\begin{abstract}
In this paper we consider a model which exhibits explicit Lorentz symmetry breaking due to the presence of a single background vector $v^{\mu}$ coupled to the gauge field. We investigate such a theory in the vicinity of a perfectly conducting plate for different configurations of $v^{\mu}$. First we consider no restrictions on the components of the background vector and we treat it perturbatively up to second order. Next, we treat $v^{\mu}$ exactly for two special cases: the first one is when it has only components parallel to the plate, and the second one when it has a single component perpendicular to the plate. For all these configurations, the propagator for the gauge field and the interaction force between the plate and a point-like electric charge are computed. Surprisingly, it is shown that the image method is valid in our model and we argue that it is a non-trivial result. We show there arises a torque on the mirror with respect to its positioning in the background field when it interacts with a point-like charge. It is a new effect with no counterpart in theories with Lorentz symmetry in the presence of a perfect mirror.
\end{abstract}

\section{Introduction}

In the last decades the searches for Lorentz violations induced by Planck scale physics became an active field of theoretical and experimental research, since it offers the hope of an observational window into quantum gravity. In order to facilitate the searches for Lorentz violation at lower energy scales, Kostelecký et al. developed the Standard Model Extension (SME) [1,2], which is a very systematic approach for the introduction of Lorentz violation in the Standard Model (SM). The SME incorporates in the SM structure all the Lorentz- and CPT-violating terms which respect renormalizability and gauge invariance. In this context, the pure photon

\footnotetext{
a e-mail: luizhenriqueunifei@yahoo.com.br

b e-mail: fbarone@unifei.edu.br
}

sector has been modified by CPT-odd [3] and CPT-even [4-7] Lorentz-violating terms.

Possible effects of Lorentz symmetry violation have been investigated in many contexts, for example, the Casimir effect [8-11], QED [12-16], hydrogen atom [17,18], noncommutative field theories [19-21], space-times with a non-trivial topology [22], gravity theory [23-28], electromagnetic wave propagation [29-32], studies of photon field quantization [33-35], effects on the classical electrodynamics [36-41], among others.

There is a gap in the literature regarding studies of Lorentzviolating theories in the presence of boundary conditions. That is a remarkable subject in any abelian gauge theory, since the experimental apparatus commonly used to test electromagnetic phenomena is, usually, surrounded by conductors. In addition, it is also important to search for physical phenomena in theories with Lorentz symmetry breaking with no counterpart in Maxwell electrodynamics, and the presence of conductors can create suitable scenarios for this kind of search.

The main purpose of this paper is to start a discussion of this subject, not yet considered in the literature, to the best of the authors' knowledge. To this end, we perform an investigation regarding some consequences of a Lorentz-violating electrodynamics due to the presence of a perfectly conducting plate. We consider the model studied in Refs. [17,36], where the Lorentz symmetry is broken in the CPT-even sector of the SME, due to the presence of a single background vector $v^{\mu}$. We consider different configurations for the background vector. First we consider the background vector with no restrictions and we treat it perturbatively up to second order. Next, we treat $v^{\mu}$ exactly for two special cases: the first one is when it has only components parallel to the plate, and the second one when it has a single component perpendicular to the plate. For all these configurations of the background vector, we obtain the propagator for the gauge field and the interaction force between the plate and a point-like 
electric charge. We also compare the interaction forces with the ones obtained in the free theory (theory without the plate) and we verify that the image method is valid in all the situations considered in this paper, which is a surprising result because Lorentz violations, usually, impose different effects in comparison with theories with Lorentz symmetry.

We show that a new effect arises when a point-like charge is placed in the vicinity of the mirror. Namely, the mirror undergoes a torque with respect to its positioning relative to the background vector. This torque tends to make the mirror perpendicular to the background vector. It is an effect due to the Lorentz symmetry breaking, with no counterpart in Maxwell electrodynamics, and it is not simply a small correction to an existing effect. Maybe this could be a way to measure some signals of possible Lorentz symmetry breaking in setups with mirrors. This fact also suggests that the subject of mirrors in gauge theories with Lorentz symmetry breaking must be well investigated.

The paper is structured as follows: in Sect. 2 we compute the propagator for the gauge field in the presence of a perfectly conducting plate considering different configurations for the background vector. We employ methods of Quantum Field Theory with boundary conditions in order to obtain the functional generator for the gauge sector. In Sect. 3, from the propagators obtained previously, we calculate the interacting forces between the conducting plate and a point-like electric charge and show that there arises a torque on the mirror. We also compare the results with the free theory in order to verify the validity of the image method. Finally, Sect. 4 is dedicated to our final remarks and conclusions.

Throughout the paper we shall deal with a model in a 3 +1 dimensional space-time and use Minkowski coordinates with the diagonal metric with signature $(+,-,-,-)$.

\section{The propagator in the presence of a conducting plate}

In this paper we consider a model for the electromagnetic field which exhibits explicit Lorentz symmetry breaking due to the presence of a single background vector; such a model is described by the following Lagrangian density $[17,36]$ :

$$
\begin{aligned}
\mathcal{L}= & -\frac{1}{4} F_{\mu \nu} F^{\mu \nu}-\frac{1}{2 \gamma}\left(\partial_{\mu} A^{\mu}\right)^{2} \\
& -\frac{1}{2} v^{\mu} v_{\nu} F_{\mu \lambda} F^{\nu \lambda}-J^{\mu} A_{\mu},
\end{aligned}
$$

where $A^{\mu}$ is the electromagnetic field, $F^{\mu \nu}=\partial^{\mu} A^{\nu}-\partial^{\nu} A^{\mu}$ is the field strength, $J^{\mu}$ is the external source, $\gamma$ is a gauge parameter and $v^{\mu}$ is the background vector, responsible for introducing the Lorentz violation in our model. As the Lorentz symmetry breaking must be very tiny, the background vector $v^{\mu}$, which is a dimensionless quantity, must be small (its components are much smaller than 1).
The model (1) is a particular case of the Lagrangian proposed in [39], where we have a tensorial parameter controlling the Lorentz symmetry breaking with a term $\sim k_{(F) \mu \nu \alpha \beta} F^{\alpha \beta} F^{\mu \nu}$. That is, when $k_{(F) \mu \nu \alpha \beta} \sim \eta_{\mu \alpha} v_{\nu} v_{\beta}-$ $\eta_{\nu \alpha} v_{\mu} v_{\beta}+\eta_{\nu \beta} v_{\mu} v_{\alpha}-\eta_{\mu \beta} v_{\nu} v_{\alpha}$ the model discussed in [39] is the same as proposed by (1).

The propagator which describes the model (1) for $\gamma=1$ is given by [36]

$$
\begin{aligned}
D^{\mu \nu}(x, y)= & -\int \frac{\mathrm{d}^{4} p}{(2 \pi)^{4}} \frac{\mathrm{e}^{-i p \cdot(x-y)}}{\left[p^{2}+(p \cdot v)^{2}\right]}\left[\eta^{\mu \nu}-\frac{v^{\mu} v^{v}}{1+v^{2}}\right. \\
& -\frac{v^{2}}{1+v^{2}} \frac{(p \cdot v)^{2}}{p^{2}} \frac{p^{\mu} p^{v}}{p^{2}} \\
& \left.+\frac{1}{1+v^{2}} \frac{(p \cdot v)}{p^{2}}\left(p^{\mu} v^{v}+v^{\mu} p^{\nu}\right)\right] .
\end{aligned}
$$

Now we have to see how the electromagnetic field is modified in the presence of a conducting surface in the model (1). To answer this question, we start by noticing that, in the model (1), the coupling between the gauge field and matter is the same as the one found in Maxwell electrodynamics, so the Lorentz forces acting on a charged particle are the same in both theories, namely, $\frac{\mathrm{d} \mathbf{p}_{p}}{\mathrm{~d} t}=q \mathbf{E}+q \mathbf{u} \times \mathbf{B}$, where $\mathbf{p}_{p}$ is the spatial component of the particle momentum, $q$ is the particle charge, $\mathbf{E}$ and $\mathbf{B}$ are the electric and magnetic fields, respectively, and $\mathbf{u}$ stands for the particle velocity.

The presence of a conducting surface in the Maxwell electrodynamics imposes a boundary condition on the gauge field in such a way that the Lorentz force on the surface must vanish. This condition is attained by taking the components of the dual field strength normal to the surface as being equal to zero. Therefore, if $n^{\mu}$ is the four-vector normal to the conducting surface, we must have $n^{\mu} F_{\mu \nu}^{*}=0$ on it, where $F_{\mu \nu}^{*}=(1 / 2) \epsilon_{\mu \nu \alpha \beta} F^{\alpha \beta}$, and $\epsilon^{\mu \nu \alpha \beta}$ stands for the Levi-Civita tensor with $\epsilon^{0123}=1$. Since the Lorentz force in the Lorentz-violating model (1) is the same as the one found in the Maxwell electrodynamics, the condition above must be satisfied by both theories.

Now, we consider the presence of a single perfectly conducting plate. We perform the calculations in a reference frame where the plate is steady. We take a coordinate system where the plate is perpendicular to the $x^{3}$ axis, located on the plane $x^{3}=a$, so that $n^{\mu}=\eta_{3}^{\mu}=(0,0,0,1)$ is the Minkowski vector perpendicular to the plate. Thus, the boundary condition on the gauge field $A^{\mu}$ becomes

$\left.F_{3 v}^{*}(x)\right|_{x^{3}=a}=\left.\epsilon_{3 v}^{\alpha \beta} \partial_{\alpha} A_{\beta}(x)\right|_{x^{3}=a}=0$,

where the sub-index means that the boundary conditions are taken on the plane $x^{3}=a$.

We must obtain the functional generator for the gauge field submitted to the boundary conditions (3). For this task 
we will use the functional formalism employed in [42]. We start by writing the functional generator as follows:

$Z_{C}[J]=\int \mathcal{D} A_{C} \mathrm{e}^{i \int \mathrm{d}^{4} x \mathcal{L}}$,

where the sub-index $C$ indicates that we are integrating out all the field configurations which satisfy the conditions (3). This restriction is attained by introducing, in the free-field functional, a functional delta that is not equal to zero only where the restrictions (3) are satisfied, as follows:

$Z_{C}[J]=\int \mathcal{D} A \delta\left[\left.F_{3 v}^{*}(x)\right|_{x^{3}=a}\right] \mathrm{e}^{i \int \mathrm{d}^{4} x \mathcal{L}}$,

where we integrate over all field configurations.

Now we use the functional Fourier representation for the delta functional,

$$
\begin{aligned}
\delta & {\left[\left.F_{3 v}^{*}(x)\right|_{x^{3}=a}\right] } \\
& =\int \mathcal{D} B \exp \left[i \int \mathrm{d}^{4} x \delta\left(x^{3}-a\right) B_{v}\left(x_{\|}\right) F_{3}^{* v}(x)\right],
\end{aligned}
$$

where $B_{v}\left(x_{\|}\right)$is an auxiliary vector field and $x_{\|}^{\mu}=$ $\left(x^{0}, x^{1}, x^{2}\right)$ means that we have only the coordinates parallel to the plate.

The auxiliary field $B_{v}\left(x_{\|}\right)$exhibits the gauge symmetry

$B_{v}^{(k)}\left(x_{\|}\right) \rightarrow B_{v}^{(k)}\left(x_{\|}\right)+\partial_{v \|} \Lambda^{(k)}\left(x_{\|}\right)$.

In order to eliminate the infinite gauge volume from the integral (6), we use the Faddeev-Popov method and the 't Hooft trick. Carrying out some manipulations, we write the functional generator as follows (for more details see Appendix A):

$Z_{C}[J]=N Z[J] \bar{Z}[J]$,

where $N$ is a constant which does not depend on the gauge fields and $Z[J]$ is the usual functional generator for the gauge field $A^{\mu}(x)$

$$
\begin{aligned}
Z[J] & =\int \mathcal{D} A \mathrm{e}^{i \int \mathrm{d}^{4} x \mathcal{L}} \\
& =Z[0] \exp \left[-\frac{i}{2} \int \mathrm{d}^{4} x \mathrm{~d}^{4} y J^{\mu}(x) D_{\mu \nu}(x, y) J^{\nu}(y)\right] .
\end{aligned}
$$

Here $\bar{Z}[J]$ is a contribution due to the vector field $B_{v}\left(x_{\|}\right)$,

$$
\begin{aligned}
\bar{Z}[J]= & \int \mathcal{D} B \exp \left[i \int \mathrm{d}^{4} x \delta\left(x^{3}-a\right) I^{v}(x) B_{v}\left(x_{\|}\right)\right] \\
& \times \exp \left[-\frac{i}{2} \int \mathrm{d}^{4} x \mathrm{~d}^{4} y \delta\left(x^{3}-a\right) \delta\left(y^{3}\right.\right. \\
& \left.-a) B_{v}\left(x_{\|}\right) W^{v \pi}(x, y) B_{\pi}\left(y_{\|}\right)\right]
\end{aligned}
$$

where we defined

$$
I^{\nu}(x)=-\int \mathrm{d}^{4} y \epsilon_{3}^{\nu \gamma \alpha}\left(\frac{\partial}{\partial x^{\gamma}} D_{\alpha \mu}(x, y)\right) J^{\mu}(y),
$$

$W^{\nu \pi}(x, y)=\epsilon_{3}{ }^{\nu \alpha \lambda} \epsilon_{3}{ }^{\pi \gamma \rho} \frac{\partial^{2} D_{\lambda \rho}(x, y)}{\partial x^{\alpha} \partial y^{\gamma}}+\frac{1}{\xi} \frac{\partial^{2} Q(x, y)}{\partial x_{v \|} \partial y_{\pi \|}}$,

and $\xi$ is a gauge fixing term related to the symmetry (7) of the auxiliary field and $Q(x, y)$ is a scalar function which must be chosen conveniently.

\subsection{The propagator in lowest order}

Since $v^{\mu}$ is very tiny, let us treat it as a small quantity and perform the calculations perturbatively up to second order in $v^{\mu}$, which is the lowest order in the background vector.

Expanding the propagator (2) up to second order in $v^{\mu}$, we obtain

$$
\begin{aligned}
D^{\mu \nu}(x, y)=- & \int \frac{\mathrm{d}^{4} p}{(2 \pi)^{4}} \frac{\mathrm{e}^{-i p \cdot(x-y)}}{p^{2}}\left[\left(1-\frac{(p \cdot v)^{2}}{p^{2}}\right) \eta^{\mu v}\right. \\
& -v^{\mu} v^{v} \\
& \left.+\frac{(p \cdot v)}{p^{2}}\left(p^{\mu} v^{v}+v^{\mu} p^{v}\right)\right]
\end{aligned}
$$

Notice that the integral (10) is Gaussian, so it can be calculated exactly. For this task it is convenient to make the following choice:

$Q(x, y)=-\int \frac{\mathrm{d}^{4} p}{(2 \pi)^{4}} \mathrm{e}^{-i p \cdot(x-y)}\left[\frac{\left(1-v_{\|}^{2}\right)}{p^{2}}-\frac{(p \cdot v)^{2}}{p^{4}}\right]$,

and work in the gauge where $\xi=1$ (for the auxiliary field in the symmetry (7)), with $v_{\|}^{\mu}=\left(v^{0}, v^{1}, v^{2}\right)$ standing for the background vector parallel to the plate. Using the propagator (13), substituting (11), (12), (14) into (10), using the fact that

$\int \frac{\mathrm{d} p^{3}}{2 \pi} \frac{\mathrm{e}^{i p^{3}\left(x^{3}-y^{3}\right)}}{p^{2}}=-\frac{i}{2 \Gamma} \mathrm{e}^{i \Gamma\left|x^{3}-y^{3}\right|}$,

$\int \frac{\mathrm{d} p^{3}}{2 \pi} \frac{\mathrm{e}^{i p^{3}\left(x^{3}-y^{3}\right)}}{p^{4}}=-\frac{1}{4 p_{\|}^{2}}\left(\frac{i}{\Gamma}+\left|x^{3}-y^{3}\right|\right) \mathrm{e}^{i \Gamma\left|x^{3}-y^{3}\right|}$,

where $p^{3}$ stands for the momentum perpendicular to the plate, and $\Gamma=\sqrt{p_{\|}^{2}}$, with $p_{\|}^{\mu}=\left(p^{0}, p^{1}, p^{2}\right)$ standing for the momentum parallel to the plate, defining the parallel metric

$\eta_{\|}^{\mu \nu}=\eta^{\mu \nu}-\eta_{3}^{\mu} \eta^{\nu 3}$, 
and performing some usual manipulations, we are able to rewrite Eq. (10) in the following form:

$$
\begin{aligned}
& \bar{Z}[J] \\
& \quad=\bar{Z}[0] \exp \left[-\frac{i}{2} \int \mathrm{d}^{4} x \mathrm{~d}^{4} y J^{\mu}(x) \bar{D}_{\mu \nu}(x, y) J^{\nu}(y)\right],
\end{aligned}
$$

where we defined the function

$$
\begin{aligned}
\bar{D}^{\mu \nu}(x, y) \\
=-\frac{i}{2} \int \frac{\mathrm{d}^{3} p_{\|}}{(2 \pi)^{3}} \mathrm{e}^{-i p_{\|} \cdot\left(x_{\|}-y_{\|}\right)}\left\{\left(\eta_{\|}^{\mu \nu}-\frac{p_{\|}^{\mu} p_{\|}^{v}}{p_{\|}^{2}}\right)\right. \\
\times\left[1+\frac{1}{2} \frac{\left(p_{\|} \cdot v_{\|}\right)^{2}}{p_{\|}^{2}}\left[i \Gamma \left(\left|x^{3}-a\right|\right.\right.\right. \\
\left.\left.+\left|y^{3}-a\right|\right)-1\right]+i\left(p_{\|} \cdot v_{\|}\right) v^{3}\left(x^{3}-y^{3}\right)+\frac{1}{2}\left(v^{3}\right)^{2} \\
\left.\times\left[i \Gamma\left(\left|x^{3}-a\right|+\left|y^{3}-a\right|\right)+1\right]\right] \\
+\frac{\left(p_{\|} \cdot v_{\|}\right)^{2}}{p_{\|}^{2}} \frac{p_{\|}^{\mu} p_{\|}^{v}}{p_{\|}^{2}}-\frac{\left(p_{\|} \cdot v_{\|}\right)}{p_{\|}^{2}} \\
\times\left[\left(v_{\|}^{v}-v^{v}\right) p_{\|}^{\mu}+\left(v_{\|}^{\mu}-v^{\mu}\right) p_{\|}^{v}\right] \\
\left.+v_{\|}^{\mu}\left(v_{\|}^{v}-v^{v}\right)-v^{\mu} v_{\|}^{v}\right\} \frac{\mathrm{e}^{i \Gamma\left(\left|x^{3}-a\right|+\left|y^{3}-a\right|\right)}}{\Gamma}
\end{aligned}
$$

with $v^{3}$ being component of the background vector perpendicular to the plate.

Substituting (17) and (9) in (8), the functional generator in the presence of a perfectly conducting plate reads

$$
\begin{aligned}
Z_{C}[J]= & Z_{C}[0] \exp \left[-\frac{i}{2} \int \mathrm{d}^{4} x \mathrm{~d}^{4} y J^{\mu}(x)\left(D_{\mu \nu}(x, y)\right.\right. \\
& \left.\left.+\bar{D}_{\mu \nu}(x, y)\right) J^{\nu}(y)\right]
\end{aligned}
$$

From Eq. (19), we can identify the propagator of the theory in the presence of a conducting plate up to second order in $v^{\mu}$, as follows:

$D_{C}^{\mu \nu}=D^{\mu \nu}(x, y)+\bar{D}^{\mu \nu}(x, y)$.

We can check the results by taking the field generated by an external source,

$A^{\beta}(x)=\int \mathrm{d}^{4} y D_{C}^{\beta \rho}(x, y) J_{\rho}(y)$.

Substituting Eq. (21) into (3) we rewrite the conducting plate condition in the following form:

$$
\begin{gathered}
\left.\int \mathrm{d}^{4} y\left[\epsilon_{3 v \alpha \beta} \frac{\partial D_{C}^{\beta \rho}(x, y)}{\partial x_{\alpha}}\right] J_{\rho}(y)\right|_{x^{3}=a}=0 \\
\left.\Rightarrow \epsilon_{3 v \alpha \beta} \frac{\partial D_{C}^{\beta \rho}(x, y)}{\partial x_{\alpha}}\right|_{x^{3}=a}=0 .
\end{gathered}
$$

Substituting Eq. (20) into (22) and then, using Eqs. (13), (15) and (18), after some simple manipulations, we can verify that the conducting plate condition (22) is satisfied.

At this point some comments are in order. The propagator (20) is composed of the sum of the free propagator (13) with the correction (18), which accounts for the presence of the conducting plate. In the limit when $v^{\mu} \rightarrow 0$ the propagator (18) reduces to the same one as that found with the Maxwell electrodynamics in the presence of a conducting plate.

\subsection{Exact propagators}

There are two special cases for which we carry out the calculations without the necessity of treating the background vector perturbatively: the first one is when $v^{\mu}$ has only components parallel to the plate, namely $v^{\mu}=v_{\|}^{\mu}$, and the second one when it has a single component which is perpendicular to the plate, namely $v^{\mu}=v^{3}$. In this subsection we obtain the exact propagator in the presence of a conducting plate in both cases mentioned above.

\subsubsection{The first case, $v^{\mu}=v_{\|}^{\mu}$}

For the case where the component of the background vector perpendicular to the plate is equal to zero, it is convenient to perform the following choice:

$Q(x, y)=-\left.\frac{1}{1+v_{\|}^{2}} \mathcal{I}(x, y)\right|_{v^{3}=0}$,

where

$\mathcal{I}(x, y)=\int \frac{\mathrm{d}^{4} p}{(2 \pi)^{4}} \frac{\mathrm{e}^{-i p \cdot(x-y)}}{\left[p^{2}+(p \cdot v)^{2}\right]}$,

and the sub-index in (23) indicates that $\mathcal{I}(x, y)$ must be evaluated only in the coordinates parallel to the plate. Using the propagator (2), substituting Eqs. (11), (12), (23) into (10), using the fact that (see Appendix B)

$\left.\mathcal{I}(x, y)\right|_{v^{3}=0}=-\frac{i}{2} \int \frac{\mathrm{d}^{3} p_{\|}}{(2 \pi)^{3}} \mathrm{e}^{-i p_{\|} \cdot\left(x_{\|}-y_{\|}\right)} \frac{1}{L} \mathrm{e}^{i L\left|x^{3}-y^{3}\right|}$,

where $L=\sqrt{p_{\|}^{2}+\left(p_{\|} \cdot v_{\|}\right)^{2}}$, and carrying out some manipulations, we can identify the correction to the propagator

$$
\begin{aligned}
& \bar{D}^{\mu \nu}(x, y)=-\frac{i}{2} \int \frac{\mathrm{d}^{3} p_{\|}}{(2 \pi)^{3}} \mathrm{e}^{-i p_{\|} \cdot\left(x_{\|}-y_{\|}\right)} \\
& \times\left(\eta_{\|}^{\mu \nu}-\frac{p_{\|}^{\mu} p_{\|}^{v}}{L^{2}}-\frac{v_{\|}^{\mu} v_{\|}^{v}}{1+v_{\|}^{2}}\right) \frac{\mathrm{e}^{i L\left(\left|x^{3}-a\right|+\left|y^{3}-a\right|\right)}}{L} .
\end{aligned}
$$

We point out that expanding Eq. (26) up to second order in $v^{\mu}$ and comparing with Eq. (18) for $v^{3}=0$, we can easily verify that these propagators become equal to each other. 


\subsubsection{The second case, $v^{\mu}=v^{3}$}

For the case where the Lorentz-violation parameter $v^{\mu}$ has only the component perpendicular to the plate, or equivalently $v_{\|}^{\mu}=0$, we make the choice

$$
Q(x, y)=-\left.\mathcal{I}(x, y)\right|_{v_{\|}^{\mu}=0},
$$

where the sub-index in (23) indicates that $\mathcal{I}(x, y)$ must be evaluated taking the coordinates of the background vector parallel to the plate equal to zero. Following the same steps employed previously and using the fact that (see Appendix B)

$$
\begin{aligned}
& \left.\mathcal{I}(x, y)\right|_{v_{\|}^{\mu}=0}=-\frac{i}{2} \frac{1}{\sqrt{1-\left(v^{3}\right)^{2}}} \int \frac{\mathrm{d}^{3} p_{\|}}{(2 \pi)^{3}} \mathrm{e}^{-i p_{\|} \cdot\left(x_{\|}-y_{\|}\right)} \\
& \times \frac{\mathrm{e}^{i \Gamma\left(\sqrt{1-\left(v^{3}\right)^{2}}\right)^{-1}\left|x^{3}-y^{3}\right|}}{\Gamma}
\end{aligned}
$$

we obtain exactly the correction to the propagator

$$
\begin{aligned}
\bar{D}^{\mu \nu}(x, y) & -\frac{i}{2} \frac{1}{\sqrt{1-\left(v^{3}\right)^{2}}} \int \frac{\mathrm{d}^{3} p_{\|}}{(2 \pi)^{3}} \mathrm{e}^{-i p_{\|} \cdot\left(x_{\|}-y_{\|}\right)} \\
& \times\left(\eta_{\|}^{\mu \nu}-\frac{p_{\|}^{\mu} p_{\|}^{v}}{p_{\|}^{2}}\right) \frac{\mathrm{e}^{i \Gamma\left(\sqrt{1-\left(v^{3}\right)^{2}}\right)^{-1}\left(\left|x^{3}-a\right|+\left|y^{3}-a\right|\right)}}{\Gamma} .
\end{aligned}
$$

In the same way, expanding Eq. (29) up to second order in $v^{\mu}$ and comparing with Eq. (18) for $v_{\|}^{\mu}=0$, we verify that these propagators become equal to each other.

\section{Charge-plate interaction}

In this section we consider the interaction between a pointlike charge and the conducting plate. We can show that the interaction energy between a conducting surface and an external source $J^{v}(x)$ is given by $[36,37,43-48]$

$E=\frac{1}{2 T} \int \mathrm{d}^{4} x \mathrm{~d}^{4} y J^{\mu}(x) \bar{D}_{\mu \nu}(x, y) J^{\nu}(y)$,

where $T$ is the time variable and the $\operatorname{limit} T \rightarrow \infty$ is implicit.

With no loss of generality and for simplicity, we choose a point-like charge placed at position perpendicular to the plate, namely $\mathbf{b}=(0,0, b)$. The external source is given by

$J^{\mu}(x)=q \eta^{\mu 0} \delta^{3}(\mathbf{x}-\mathbf{b})$,

where the parameter $q$ is a coupling constant between the field and the delta function, which can be seen as the electric charge.

\subsection{Results in lowest order}

Substituting Eqs. (31) and (18) in (30), carrying out the integrals in $\mathrm{d}^{3} \mathbf{x}, \mathrm{d}^{3} \mathbf{y}, \mathrm{d} x^{0}, \mathrm{~d} p^{0}, \mathrm{~d} y^{0}$ and then performing some manipulations, we obtain

$$
\begin{aligned}
E_{P C} \cong- & \frac{q^{2}}{16 \pi^{2}} \int \mathrm{d}^{2} \mathbf{p}_{\|} \frac{\mathrm{e}^{-2 R \sqrt{\mathbf{p}_{\|}^{2}}}}{\sqrt{\mathbf{p}_{\|}^{2}}}\left[1-\left(v^{0}\right)^{2}\right. \\
& +\frac{1}{2}\left(1+2 R \sqrt{\mathbf{p}_{\|}^{2}}\right) \frac{\left(\mathbf{p}_{\|} \cdot \mathbf{v}_{\|}\right)^{2}}{\mathbf{p}_{\|}^{2}} \\
& \left.+\frac{1}{2}\left(v^{3}\right)^{2}\left(1-2 R \sqrt{\mathbf{p}_{\|}^{2}}\right)\right]
\end{aligned}
$$

where $R=|a-b|$ is the distance between the plate and the charge. The sub-index $P C$ means that we have the interaction energy between the conducting plate and the charge.

Equation (32) can be simplified by using polar coordinates and integrating out the angular variables,

$$
\begin{aligned}
E_{\mathrm{PC}} \cong & -\frac{q^{2}}{16 \pi}\left[2\left(1-\left(v^{0}\right)^{2}+\frac{1}{2}\left(v^{3}\right)^{2}+\frac{1}{4} \mathbf{v}_{\|}^{2}\right)\right. \\
& \times \int_{0}^{\infty} \mathrm{d} p \mathrm{e}^{-2 R p}+R\left[\mathbf{v}_{\|}^{2}-2\left(v^{3}\right)^{2}\right] \\
& \left.\times \int_{0}^{\infty} \mathrm{d} p p \mathrm{e}^{-2 R p}\right]
\end{aligned}
$$

Using the fact that

$\int_{0}^{\infty} \mathrm{d} p \mathrm{e}^{-2 R p}=\frac{1}{2 R}, \int_{0}^{\infty} \mathrm{d} p p \mathrm{e}^{-2 R p}=\frac{1}{4 R^{2}}$,

the interaction energy between the plate and the charge, up to second order in $v^{\mu}$, reads

$E_{\mathrm{PC}}=-\frac{q^{2}}{16 \pi R}\left[1-\left(v^{0}\right)^{2}+\frac{1}{2} \mathbf{v}_{\|}^{2}\right]$.

Equation (35) is a perturbative result and gives the interaction energy between a point-like charge and a conducting plate for the model (1). The first term on the right hand side is the plate-charge interaction obtained in Maxwell electrodynamics, where the image method is valid; it does not involve the Lorentz-violation parameter $v^{\mu}$. The second and third terms are corrections due to the Lorentz symmetry breaking.

Let us make a cautious analysis of Eq. (35).

First we focus only on the dependence of the energy (35) with respect to the distance $R$. In this case, Eq. (35) exhibits the same behavior found in the usual Coulomb interaction between the point-like charge and its image charge. The corresponding interaction force between the point-like charge and the plate is given by

$F_{\mathrm{PC}}=-\frac{\partial E_{\mathrm{PC}}}{\partial R}=-\frac{q^{2}}{16 \pi R^{2}}\left[1-\left(v^{0}\right)^{2}+\frac{1}{2} \mathbf{v}_{\|}^{2}\right]$. 
Equation (36) is the usual Coulombian interaction between an effective charge $q \sqrt{1-\left(v^{0}\right)^{2}+(1 / 2) \mathbf{v}_{\|}^{2}}$ and its image, placed at a distance $2 R$ apart. Thus, the Lorentz violation generates a correction to the charge $q$ in this specific setup.

In Eq. (18) of Ref. [36] we have the interaction force between two point-like charges for the model (1) up to second order in $v^{\mu}$. From this expression, we can obtain the interaction force for the special case where we have two opposite charges, $\sigma_{1}=q$ and $\sigma_{2}=-q$, placed at a distance $2 R$ apart. In this specific situation, this energy turns out to be equivalent to Eq. (36).

It is interesting to notice that the image method is valid for the Lorentz-violation theory (1) up to second order in $v^{\mu}$ for the conducting plate condition (3).

Now we consider the dependence of the energy (35) as a function of the positioning of the mirror with respect to the background vector. Designating by $\alpha$ the angle between the normal to the mirror and the background vector $\mathbf{v}$ (defined for $0 \leq \alpha \leq \pi$ ), we have $\mathbf{v}_{\|}^{2}=\mathbf{v}^{2} \cos ^{2}(\alpha)$ and the energy (35) reads

$E_{\mathrm{PC}}=-\frac{q^{2}}{16 \pi R}\left[1-\left(v^{0}\right)^{2}+\frac{1}{2} \mathbf{v}^{2} \cos ^{2}(\alpha)\right]$.

In addition to the force between the charge and the mirror, the energy (37) also leads to a torque acting on the mirror, as follows:

$\tau_{\mathrm{PC}}=-\frac{\partial E_{\mathrm{PC}}}{\partial \alpha}=-\frac{q^{2} \mathbf{v}^{2}}{32 \pi R} \sin (2 \alpha)$.

This torque depends just on the angle between the normal to the plate and the background vector $\mathbf{v}$, as well as on the distance between the charge and the plate, $R$, but it does not depend on the position of the charge. This is a new effect with no counterpart in Maxwell electrodynamics in the presence of a conducting surface.

Maybe this torque could be a way to measure possible effects of Lorentz symmetry breaking in setups with mirrors. Although it is a small effect, in order $\mathbf{v}^{2}$, there is no other similar effect found in the standard theories with Lorentz symmetry. So the torque (38) is not simply a very small correction to a given effect, but it is an authentic effect by itself which arises in a Lorentz-violating scenario.

In Fig. 1 we have a plot for the torque (38) as a function of $\alpha$, which exhibits three zeros, corresponding to the extreme values for the energy (37). For $\alpha=0$, and $\pi$, we have a minimum for the energy, so these are points of stable equilibrium. For $\alpha=\pi / 2$, the energy has a maximum, so it is an unstable equilibrium point. In the region $0 \leq \alpha \leq \pi / 2$ the torque is negative, which means that it is a restoring torque which tends to diminish the angle $\alpha$ between the normal to the plate and the background vector. For $\alpha=\pi / 4$, this torque has a minimum. Thus, in the region $0 \leq \alpha \leq \pi / 2$ the mirror

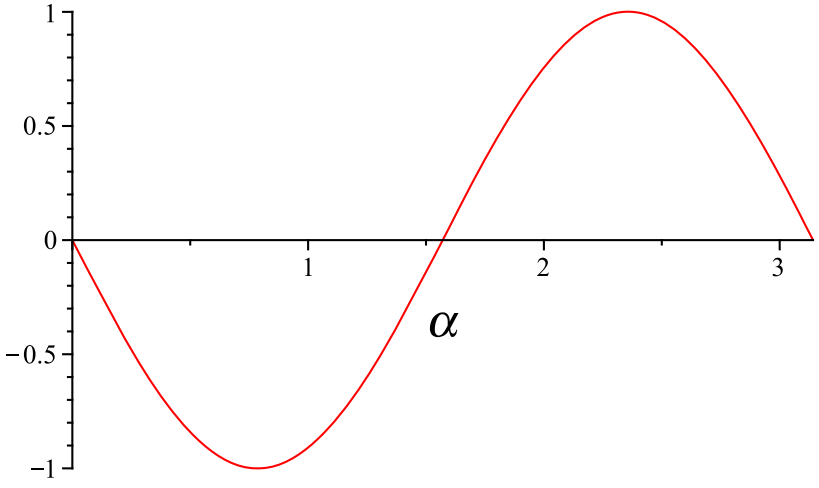

Fig. 1 Plot for $\tau_{P C}$, with $q^{2} \mathbf{v}^{2} /(32 \pi R)=1$, as a function of $\alpha$. The torque vanishes for $\alpha=0, \pi / 2, \pi$ and two extrema values for $\alpha=$ $\pi / 4,3 \pi / 4$

tends to be normal to the background vector. In the region $\pi / 2 \leq \alpha \leq \pi$ the torque is always positive, which means that the angle between the normal to the mirror and the background vector tends to increase till its maximum value $\pi$, where the mirror is, again, normal to the background vector. In this region, the maximum value for the torque occurs for $\alpha=3 \pi / 4$.

For $\alpha=\pi / 2$, where we have an unstable equilibrium, the mirror is parallel to the background vector. Any small change in this configuration shall make the mirror rotate to one of its stable equilibrium points $\alpha=0, \pi$.

\subsection{Exact results}

\subsubsection{The first case, $v^{\mu}=v_{\|}^{\mu}$}

Substituting Eqs. (31) and (26) in (30), following the same steps as previously, we obtain

$E_{\mathrm{PC}}=-\frac{q^{2}}{16 \pi^{2}} \frac{1-\mathbf{v}_{\|}^{2}}{1+v_{\|}^{2}} \int \mathrm{d}^{2} \mathbf{p}_{\|} \frac{\mathrm{e}^{-2 R \sqrt{\mathbf{p}_{\|}^{2}-\left(\mathbf{p}_{\|} \cdot \mathbf{v}_{\|}\right)^{2}}}}{\sqrt{\mathbf{p}_{\|}^{2}-\left(\mathbf{p}_{\|} \cdot \mathbf{v}_{\|}\right)^{2}}}$.

In Eq. (39), performing a change in the integration variables similar to the one we have made in Appendix B, and then using polar coordinates, we obtain

$E_{\mathrm{PC}}=-\frac{q^{2}}{16 \pi R} \frac{\sqrt{1-\mathbf{v}_{\|}^{2}}}{1+v_{\|}^{2}}$.

Equation (40) gives the exact expression for the interaction energy between a point-like charge and a conducting plate for the special case where the background vector has only the parallel components to the plate.

The interaction force reads

$F_{\mathrm{PC}}=-\frac{\partial E_{\mathrm{PC}}}{\partial R}=-\frac{q^{2}}{16 \pi R^{2}} \frac{\sqrt{1-\mathbf{v}_{\|}^{2}}}{1+v_{\|}^{2}}$, 
which is the usual result found in Maxwell electrodynamics with an effective charge $q \sqrt{\frac{\left(1-\mathbf{v}_{\|}^{2}\right)^{1 / 2}}{1+v_{\|}^{2}}}$.

Expanding Eq. (41) up to second order in $v^{\mu}$ we obtain the result (36). This occurs because Eq. (36) does not depend on the component of the background vector perpendicular to the plate $v^{3}$.

In Eq. (17) of Ref. [36] we have the exact interaction force between two point-like charges. For the special situation where $v^{3}=0, \sigma_{1}=q, \sigma_{2}=-q$ and $|\mathbf{a}|=2 R$, this result turns out to be equivalent to Eq. (41) above. Thus, for this special case $\left(v^{3}=0\right)$, the image method is valid for the conducting plate condition (3).

\subsubsection{The second case, $v^{\mu}=v^{3}$}

Substituting Eqs. (31) and (29) in (30) and performing some simple manipulations, we verify that the interaction force between a point-like charge and a conducting plate for the special case where the background vector has only the perpendicular component to the plate is equivalent to the result obtained in Maxwell electrodynamics, whose corresponding force is given by

$F_{\mathrm{PC}}=-\frac{q^{2}}{16 \pi R^{2}}$

Therefore, we have no effect due to the Lorentz symmetry breaking in this case.

In the same way, taking $v_{\|}^{\mu}=0, \sigma_{1}=q, \sigma_{2}=-q$ and $|\mathbf{a}|=2 R$ in Eq. (17) of Ref. [36], we obtain the result (42). Thus, the image method is valid for the case where $v^{\mu}=\left(0,0,0, v^{3}\right)$.

It is important to mention that the validity of the image method in a Lorentz-violating scenario, as shown in this subsection, is a highly non-trivial result, which suggests that the presence of conductors in Lorentz-violating scenarios is a subject which deserves more investigation.

This very non-intuitive result can be more evinced if we consider a similar situation in a gauge theory which exhibits Lorentz symmetry; the Podolsky-Lee-Wick electrodynamics [49-53]. In this case, it is shown that the image method is not valid $[43,54]$, in spite of the gauge and Lorentz symmetries.

\section{Conclusions and perspectives}

In this paper some consequences of the Lorentz-violation theory (1) due to the presence of a single perfectly conducting plate were studied. We argued what the role is of a perfectly conducting plate in a Lorentz-violation theory and obtained the functional generator for the gauge sector. We considered different configurations of the background vector. First we took into account all the components of the background vector and we treated it perturbatively up to second order. Next, we treated the theory exactly for two special cases: the first one, when the background vector has only components parallel to the plate and the second one, when it has a single component which is perpendicular to the plate. For all these configurations of the background vector, we obtained the propagator for the gauge field and the interaction force between the plate and a point-like electric charge. We showed that the image method is valid in the considered theory, which exhibits Lorentz symmetry violation, which is a non-trivial result.

We also showed that a new effect arises from the obtained results, a torque acting on the mirror according to its positioning with respect to the background vector. This torque tends to make the mirror normal to the background vector and, maybe, it could be a way to measure possible effects of Lorentz symmetry breaking in systems with the presence of mirrors; it is not simply a very small correction to a given effect, but it is an authentic effect by itself which arises in a scenario with Lorentz symmetry breaking.

An interesting extension of this work would be the investigation of the interaction force between two parallel plates (Casimir effect) [55] in the model considered in this paper, which would be a much more complicated problem.

We hope that this paper could be a start in the discussion regarding the role of conductors in scenarios with Lorentz symmetry breaking.

Acknowledgements L.H.C. Borges thanks to São Paulo Research Foundation (FAPESP) under the grant 2016/11137-5 for financial support. F.A. Barone thanks to $\mathrm{CNPq}$ (Brazilian agency) under the grant 311514/2015-4 for financial support.

Open Access This article is distributed under the terms of the Creative Commons Attribution 4.0 International License (http://creativecomm ons.org/licenses/by/4.0/), which permits unrestricted use, distribution, and reproduction in any medium, provided you give appropriate credit to the original author(s) and the source, provide a link to the Creative Commons license, and indicate if changes were made.

Funded by SCOAP . $^{3}$

\section{Appendix A: functional generator}

In this appendix we obtain Eq. (8). First we must eliminate the infinite gauge volume from the integral (6) due to the gauge invariance (7). In order to accomplish this task we use the Faddeev-Popov trick, fixing the covariant gauge,

$F\left[B_{v}\left(x_{\|}\right)\right]=\partial_{\|}^{v} B_{v}\left(x_{\|}\right)=f\left(x_{\|}\right)$,

where $f\left(x_{\|}\right)$is an arbitrary function. Since the FaddeevPopov determinant is independent of the field $B_{v}^{k}\left(x_{\|}\right)$, it does not contribute to the integral. Thus, we can write Eq. (6) as follows: 


$$
\begin{gathered}
\delta\left[\left.F_{3 v}^{*}(x)\right|_{x^{3}=a}\right] \sim \int \mathcal{D} B \delta\left[F\left[B_{v}\left(x_{\|}\right)\right]-f\left(x_{\|}\right)\right] \\
\quad \times \exp \left[i \int \mathrm{d}^{4} x \delta\left(x^{3}-a\right) B_{v}\left(x_{\|}\right) F_{3}^{* v}(x)\right] .
\end{gathered}
$$

Now we integrate by parts the argument of the exponential and use the 't Hooft trick, multiplying both sides of Eq. (A2) by a convergent functional of $f\left(x_{\|}\right)$and integrating over $f\left(x_{\|}\right)$,

$$
\begin{gathered}
\delta\left[\left.F_{3 v}^{*}(x)\right|_{x^{3}=a}\right]=N \int \mathcal{D} f \int \mathcal{D} B \delta\left[F\left[B_{v}\left(x_{\|}\right)\right]-f\left(x_{\|}\right)\right] \\
\times \exp \left[-i \int \mathrm{d}^{4} x \delta\left(x^{3}-a\right) A_{\beta}(x) \epsilon_{3}^{v \alpha \beta} \partial_{\alpha} B_{v}\left(x_{\|}\right)\right] \\
\times \exp \left[-\frac{i}{2 \xi} \int \mathrm{d}^{4} x \mathrm{~d}^{4} y \delta\left(x^{3}\right.\right. \\
\left.-a) f\left(x_{\|}\right) Q(x, y) f\left(y_{\|}\right) \delta\left(y^{3}-a\right)\right],
\end{gathered}
$$

where $Q(x, y)$ is an arbitrary function, $N$ is a constant, and $\xi$ is an arbitrary gauge constant.

Performing the functional integral in $f$ and two integrations by parts in Eq. (A3), we obtain

$$
\begin{aligned}
\delta\left[\left.F_{3 v}^{*}(x)\right|_{x^{3}=a}\right] & =N \int \mathcal{D} B \exp \left[-i \int \mathrm{d}^{4} x \delta\left(x^{3}-a\right)\right. \\
& \left.\times A_{\beta}(x) \epsilon_{3}{ }^{\nu \alpha \beta} \partial_{\alpha} B_{v}\left(x_{\|}\right)\right] \\
& \times \exp \left[-\frac{i}{2 \xi} \int \mathrm{d}^{4} x \mathrm{~d}^{4} y \delta\left(x^{3}-a\right)\right. \\
& \left.\times B_{\mu}\left(x_{\|}\right) \frac{\partial^{2} Q(x, y)}{\partial x_{\mu \|} \partial y_{\nu \|}} \delta\left(y^{3}-a\right) B_{v}\left(x_{\|}\right)\right] .
\end{aligned}
$$

Substituting Eq. (A4) in (5) we have

$$
\begin{aligned}
Z_{C}[J] & N \int \mathcal{D} A \mathcal{D} B \mathrm{e}^{i \int \mathrm{d}^{4} x \mathcal{L}} \exp \left[-i \int \mathrm{d}^{4} x \delta\left(x^{3}-a\right)\right. \\
& \left.\times A_{\beta}(x) \epsilon_{3}^{\nu \alpha \beta} \partial_{\alpha} B_{v}\left(x_{\|}\right)\right] \\
& \times \exp \left[-\frac{i}{2 \xi} \int \mathrm{d}^{4} x \mathrm{~d}^{4} y \delta\left(x^{3}-a\right)\right. \\
& \left.\times B_{\mu}\left(x_{\|}\right) \frac{\partial^{2} Q(x, y)}{\partial x_{\mu \|} \partial y_{v \|}} \delta\left(y^{3}-a\right) B_{v}\left(x_{\|}\right)\right]
\end{aligned}
$$

In the first exponential we have only the $A^{\mu}$ field, and in the third one only the presence of $B^{\mu}$. The second exponential contains a coupling between $A$ and $B$. In order to decouple the fields $A$ and $B$ we perform the translation

$$
\begin{aligned}
& A^{\beta}(x) \rightarrow A^{\beta}(x) \\
& +\int \mathrm{d}^{4} y D_{\alpha}^{\beta}(x, y) \delta\left(y^{3}-a\right) \epsilon_{3}^{v \gamma \alpha} \partial_{\gamma} B_{v}(y),
\end{aligned}
$$

which allows us to write Eq. (A5) as follows:

$Z_{C}[J]=N Z[J] \bar{Z}[J]$,

where $Z[J]$ is given by Eq. (9) and $\bar{Z}[J]$ by Eq. (10).

\section{Appendix B: Integral}

In this appendix we compute the following integral:

$\mathcal{I}(x, y)=\int \frac{\mathrm{d}^{4} p}{(2 \pi)^{4}} \frac{\mathrm{e}^{-i p \cdot(x-y)}}{\left[p^{2}+(p \cdot v)^{2}\right]}$.

In order to put $\mathcal{I}(x, y)$ in an appropriate form, we have to carry out a change of the integration variables. We split the four-vector momentum $p^{\mu}$ into two parts, one parallel, $p_{p}^{\mu}$, and the other normal, $p_{n}^{\mu}$, to the Lorentz-violation parameter $v^{\mu}$, namely

$$
\begin{aligned}
p^{\mu} & =p_{n}^{\mu}+p_{p}^{\mu}, \quad p_{p}^{\mu}=\left(\frac{v \cdot p}{v^{2}}\right) v^{\mu}, \quad p_{n}^{\mu} \\
& =p^{\mu}-\left(\frac{v \cdot p}{v^{2}}\right) v^{\mu}
\end{aligned}
$$

where $p_{n} \cdot v=0$ and $(p \cdot v)^{2}=p_{p}^{2} v^{2}$. Now we define the four-vector $q^{\mu}$

$$
\begin{aligned}
q^{\mu} & =p_{n}^{\mu}+p_{p}^{\mu} \sqrt{1+v^{2}} \\
& =p^{\mu}+\left(\frac{v \cdot p}{v^{2}}\right)\left(\sqrt{1+v^{2}}-1\right) v^{\mu}
\end{aligned}
$$

With definitions (B2) and (B3), we have

$$
\begin{aligned}
p_{p}^{\mu} & =\frac{(v \cdot q)}{v^{2}} \frac{v^{\mu}}{\sqrt{1+v^{2}}}, \quad p_{n}^{\mu}=q^{\mu}-\frac{(v \cdot q)}{v^{2}} v^{\mu} \\
& \Rightarrow p^{\mu}=q^{\mu}+\frac{(v \cdot q)}{v^{2}}\left(\frac{1}{\sqrt{1+v^{2}}}-1\right) v^{\mu} \\
q^{2} & =p^{2}+(p \cdot v)^{2} .
\end{aligned}
$$

With the aid of the definition

$b^{\mu}=\left(x^{\mu}-y^{\mu}\right)+\left(\frac{1-\sqrt{1+v^{2}}}{\sqrt{1+v^{2}}}\right)\left(\frac{v \cdot(x-y)}{v^{2}}\right) v^{\mu}$

and Eq. (B4), we have

$p \cdot(x-y)=b \cdot q$.

The Jacobian of the transformation from $p^{\mu}$ to $q^{\mu}$ can be calculated from Eq. (B4),

$\operatorname{det}\left[\frac{\partial p^{\mu}}{\partial q_{v}}\right]=-\frac{1}{\sqrt{1+v^{2}}}$ 
Substituting Eqs. (B5), (B7), (B8) in (B1), we obtain

$$
\begin{aligned}
\mathcal{I}(x, y) & =-\frac{1}{\sqrt{1+v^{2}}} \int \frac{\mathrm{d}^{4} q}{(2 \pi)^{4}} \frac{\mathrm{e}^{-i b \cdot q}}{q^{2}} \\
& =-\frac{1}{\sqrt{1+v^{2}}} \int \frac{\mathrm{d}^{3} q_{\|}}{(2 \pi)^{3}} \mathrm{e}^{-i b_{\|} \cdot q_{\|}} \int \frac{\mathrm{d} q^{3}}{2 \pi} \frac{\mathrm{e}^{i q^{3} b^{3}}}{q^{2}} .
\end{aligned}
$$

The last integral in the expression above is given by

$\int \frac{\mathrm{d} q^{3}}{2 \pi} \frac{\mathrm{e}^{i q^{3} b^{3}}}{q^{2}}=-\frac{i}{2 L} \mathrm{e}^{i L\left|b^{3}\right|}$,

where $L=\sqrt{q_{\|}^{2}}$ or, from Eq. (B3), $L=\sqrt{p_{\|}^{2}+\left(p_{\|} \cdot v_{\|}\right)^{2}}$ and $b^{3}$ is found by taking $\mu=3$ in (B6), as follows:

$b^{3}=\left(x^{3}-y^{3}\right)+\left(\frac{1-\sqrt{1+v^{2}}}{\sqrt{1+v^{2}}}\right)\left(\frac{v \cdot(x-y)}{v^{2}}\right) v^{3}$.

The second integral in Eq. (B9) is given by

$\int \frac{\mathrm{d}^{3} q_{\|}}{(2 \pi)^{3}} \mathrm{e}^{-i b_{\|} \cdot q_{\|}}=-\sqrt{1+v_{\|}^{2}} \int \frac{\mathrm{d}^{3} p_{\|}}{(2 \pi)^{3}} \mathrm{e}^{-i p_{\|} \cdot\left(x_{\|}-y_{\|}\right)}$,

where we used Eqs. (B7) and (B8).

Collecting terms, we write

$\mathcal{I}(x, y)=-\frac{i}{2} \sqrt{\frac{1+v_{\|}^{2}}{1+v^{2}}} \int \frac{\mathrm{d}^{3} p_{\|}}{(2 \pi)^{3}} \mathrm{e}^{-i p_{\|} \cdot\left(x_{\|}-y_{\|}\right)} \frac{1}{L} \mathrm{e}^{i L\left|b^{3}\right|}$.

Finally, taking $v^{3}=0$ in Eq. (B13) we obtain Eq. (25). In the same way, taking $v_{\|}^{\mu}=0$ in Eq. (B13), we obtain Eq. (28).

\section{References}

1. D. Colladay, V.A. Kostelecký, Phys. Rev. D 55, 6760 (1997)

2. D. Colladay, V.A. Kostelecký, Phys. Rev. D 58, 116002 (1998)

3. S. Carroll, G. Field, R. Jackiw, Phys. Rev. D 41, 1231 (1990)

4. V.A. Kostelecký, M. Mewes, Phys. Rev. Lett. 87, 251304 (2001)

5. V.A. Kostelecký, M. Mewes, Phys. Rev. D 66, 056005 (2002)

6. V.A. Kostelecký, M. Mewes, Phys. Rev. Lett. 97, 140401 (2006)

7. V.A. Kostelecký, M. Mewes, Phys. Rev. D 80, 015020 (2009)

8. M. Frank, I. Turan, Phys. Rev. D 74, 033016 (2006)

9. A.F. Santos, F.C. Khanna, Phys. Lett. B 762, 283 (2016)

10. L. M. Silva, H. Belich, J. A. Helayël-Neto, arXiv:1605.02388 (2016)

11. A. Martn-Ruiz, C.A. Escobar, Phys. Rev. D 94, 076010 (2016)

12. G. P. de Brito, J. T. Guaitolini Junior, D. Kroff, P. C. Malta, C. Marques, arXiv:1605.08059 (2016)

13. F.R. Klinkhamer, M. Schreck, Nuc. Phys. B 848, 90 (2011)

14. D. Colladay, V.A. Kostelecký, Phys. Lett. B 511, 209 (2001)

15. M.A. Hohensee, R. Lehnert, D.F. Phillips, R.L. Walsworth, Phys. Rev. D 80, 036010 (2009)
16. V.A. Kostelecký, A.G.M. Pickering, Phys. Rev. Lett. 91, 031801 (2003)

17. L.H.C. Borges, F.A. Barone, Eur. Phys. J. C 76, 64 (2016)

18. H. Belich, T. Costa-Soares, M.M. Ferreira Jr., J.A. Helayël-Neto, F.M.O. Moucherek, Phys. Rev. D 74, 065009 (2006)

19. I. Mocioiu, M. Pospelov, R. Roiban, Phys. Lett. B 489, 390 (2000)

20. C.E. Carlson, C.D. Carone, R.F. Lebed, Phys. Lett. B 518, 201 (2001)

21. A. Anisimov, T. Banks, M. Dine, M. Graesser, Phys. Rev. D 65, 085032 (2002)

22. F.R. Klinkhamer, Nucl. Phys. B 535, 233 (1998)

23. R. Bluhm, S.H. Fung, V.A. Kostelecký, Phys. Rev. D 77, 065020 (2008)

24. R. Jackiw, S.Y. Pi, Phys. Rev. D 68, 104012 (2003)

25. Z. Berezhiani, D. Comelli, F. Nesti, L. Pilo, Phys. Rev. Lett. 99, 131101 (2007)

26. V.A. Kostelecký, R. Potting, Phys. Rev. D 79, 065018 (2009)

27. J.L. Boldo, J.A. Helayel-Neto, L.M. de Moraes, C.A.G. Sasaki, V.V.J. Otoya, Phys. Lett. B 689, 112 (2010)

28. A.F. Ferrari, M. Gomes, J.R. Nascimento, E. Passos, A.Y. Petrov, A.J. da Silva, Phys. Lett. B 652, 174-180 (2007)

29. L.H.C. Borges, A.G. Dias, A.F. Ferrari, J.R. Nascimento, A.Y. Petrov, Phys. Lett. B 756, 332 (2016)

30. B.T. Agostini, F.A. Barone, F.E. Barone, P.A. Gaete, J.A. HelayëlNeto, Phys. Lett. B 708, 212 (2012)

31. R. Casana, M.M. Ferreira Jr., R.P.M. Moreira, Eur. Phys. J. C 72, 2070 (2012)

32. G. Gubitosi, G. Genovese, G. Amelino-Camelia, A. Melchiorri, Phys. Rev. D 82, 024013 (2010)

33. D. Colladay, P. McDonald, R. Potting, Phys. Rev. D 89, 085014 (2014)

34. R. Casana, M.M. Ferreira Jr., F.E.P. dos Santos, Phys. Rev. D 90, 105025 (2014)

35. R. Casana, M.M. Ferreira Jr., F.E.P. dos Santos, Phys. Rev. D 94, 125011 (2016)

36. L.H.C. Borges, F.A. Barone, J.A. Helayël-Neto, Eur. Phys. J. C 74, $2937(2014)$

37. L.H.C. Borges, A.F. Ferrari, F.A. Barone, Eur. Phys. J. C 76, 599 (2016)

38. H. Belich, M.M. Ferreira, J.A. Helayël-Neto, M.T.D. Orlando, Phys. Rev. D 68, 025005 (2003)

39. Q.G. Bailey, V.A. Kkostelecký, Phys. Rev. D 70, 076006 (2004)

40. R. Casana, M.M. Ferreira Jr., A.R. Gomes, P.R.D. Pinheiro, Eur. Phys. J. C 62, 573-578 (2009)

41. R. Casana, M.M. Ferreira Jr., C.E.H. Santos, Phys. Rev. D 78, $105014(2008)$

42. M. Bordag, D. Robaschik, E. Wieczorek, Quantum field theoretic treatment of the casimir effect. Ann. Phys. 165, 192-213 (1985)

43. F.A. Barone, A.A. Nogueira, Eur. Phys. J. C 75, 339 (2015)

44. F.A. Barone, G. Flores-Hidalgo, Phys. Rev. D 78, 125003 (2008)

45. F.A. Barone, G. Flores-Hidalgo, Braz. J. Phys. 40, 188 (2010)

46. G.T. Camilo, F.A. Barone, F.E. Barone, Phys. Rev. D 87, 025011 (2013)

47. F.A. Barone, G. Flores-Hidalgo, A.A. Nogueira, Phys. Rev. D 88, 105031 (2013)

48. F.A. Barone, F.E. Barone, Phys. Rev. D 89, 065020 (2014)

49. Boris Podolsky, Phys. Rev. D 62, 68 (1942)

50. Boris Podolsky, Chihiro Kikuchi, Phys. Rev. D 65, 228 (1944)

51. Boris Podolsky, Philip Schwed, Rev. Mod. Phys. 29, 40 (1948)

52. T.D. Lee, G.C. Wick, Nucl. Phys. B 9, 209 (1969)

53. T.D. Lee, G.C. Wick, Phys. Rev. D 2, 103 (1970)

54. F.A. Barone, G. Flores-Hidalgo, A.A. Nogueira, Phys. Rev. D 91, 027701 (2015)

55. L.H.C. Borges et al, Casimir energy in a Lorentz violating scenario (to be submitted) 